\title{
Efeito do jejum e da administração de insulina sobre os parâmetros metabólicos de ovelhas em confinamento
}

\author{
Effect of fasting and insulin administration on metabolic parameters of confined sheep
}

\author{
Augusto Schneider ${ }^{1}$, Elizabeth Schwegler ${ }^{2}$, Maikel Alan Goulart ${ }^{2}$, Talita Bandeira Roos ${ }^{2}$, Viviane Rhorig \\ Rabassa ${ }^{2}$, Francisco Augusto Burklet Del Pino ${ }^{3}$, Luiz Francisco Machado Pfeifer ${ }^{4}$ \& Marcio Nunes \\ Corrêa $^{2}$
}

\begin{abstract}
RESUMO
Os parâmetros bioquímicos sangüíneos variam amplamente, sendo os níveis de glicose, uréia e corpos cetônicos utilizados para o diagnóstico de condições patológicas em ovinos, e o aumento do conhecimento das respostas metabólicas, importante para a otimização de programas de nutrição. O objetivo do presente estudo foi avaliar a resposta metabólica de ovelhas confinadas submetidas ao jejum, tratamento com insulina e ao jejum associado ao tratamento com insulina. Para a realização deste experimento foram utilizadas 8 ovelhas mantidas em confinamento. As ovelhas foram divididas em quatro grupos, sendo estes: grupo controle, que não recebeu tratamento; grupo insulina, que recebeu a administração de insulina durante os 3 dias do experimento; grupo jejum, que foi submetido a jejum por 3 dias e o grupo jejum e insulina, que foi submetido ao jejum e administração de insulina. Foram realizadas coletas de sangue durante os 3 dias do experimento para análise dos níveis de glicose e uréia, e insulina no último dia. No final do terceiro dia do experimento, também foi realizada análise dos níveis de proteína total, albumina, beta-hidroxibutirato, triacilglicerídeos, colesterol, magnésio, fósforo e fator de crescimento semelhante a insulina tipo I. Não foram observadas alterações clínicas durante o período experimental. Não houve variações relevantes nos marcadores analisados entre os grupos, o que demonstra uma grande capacidade de adaptação dos animais às variações nutricionais de curto prazo, as quais os animais foram submetidos neste experimento. Portanto, ovelhas confinadas adaptadas a um padrão alimentar, são capazes de resistir a variações de curto período no padrão alimentar, não apresentando mudanças consideráveis nos níveis dos marcadores metabólicos estudados.
\end{abstract}

Descritores: jejum, insulina, metabolismo, ovinos.

\section{ABSTRACT}

The biochemical parameters vary widely, however the levels of glucose, urea and ketone bodies are used for diagnostic of pathological conditions in sheep, the increase knowledge of the metabolic pathways is also important for the optimization of nutritional programs. The aim of the present trial was to evaluate the metabolic response of confined sheep submitted to fasting, insulin treatment and fasting plus insulin treatment. For this experiment 8 sheep kept in a confinement were used. The sheep were divided in four groups: control group, that didn't receive any treatment; insulin group, that receive insulin administration among 3 days; fasting group, that was submitted to fasting for 3 days and fasting plus insulin group, that was submitted to fasting and also receive insulin. The blood collections were realized during the 3 days of experiment to evaluate the glucose and urea levels and insulin in the last day. At the end of the third day of the experiment were evaluated the levels of protein, albumin, beta-hidroxibutirate, triacylglycerol, cholesterol, magnesium, phosphorus and insulin-like growth factor type I. There weren't observed any clinical signs during the experiment. There weren't significant variations in the levels of the markers studied between groups, evidencing the great ability of the animals to adapt to short term nutritional variation imposed at this experiment. Thus, confined sheep adapted to a feeding pattern are able to resist to short term nutritional variability and didn't show any considerable change in the levels of the metabolic markers analyzed.

Key words: fasting, insulin, metabolism, sheep. 


\section{INTRODUÇÃO}

Os parâmetros bioquímicos sangüíneos variam amplamente, sendo os níveis de glicose, uréia e corpos cetônicos comumente utilizados para o diagnóstico de condições patológicas em ovinos [6]. O aumento de conhecimento das respostas metabólicas também é importante para possibilitar a otimização de programas de nutrição animal [2], sendo de interesse o entendimento de como os animais adaptam-se a deficiências na dieta [1].

A insulina é um importante hormônio metabólico que atua no transporte de glicose e aminoácidos para o interior da célula e favorecendo a síntese de proteínas, glicogênio e triglicerídeos [4], sendo secretada em resposta à superalimentação [2]. Em períodos de jejum, há uma redução da secreção de insulina e aumento de glucagon, que possui efeito inverso ao da insulina [4]. Devido a este papel central no metabolismo, a avaliação do nível de insulina permite entender como os animais estão respondendo a determinada condição nutricional.

Baseado nestas observações, o objetivo do presente estudo foi avaliar a resposta metabólica de ovelhas confinadas submetidas ao jejum, tratamento com insulina e ao jejum associado ao tratamento com insulina.

\section{MATERIAIS E MÉTODOS}

Para a realização deste experimento foram utilizadas 8 ovelhas (Texel x Corriedale), não prenhes, mantidas em sistema de confinamento, recebendo concentrado ( $1 \%$ do peso vivo) e feno de tifton. Previamente ao início do experimento, as ovelhas foram divididas em quatro grupos, sendo estes: grupo controle ( $\mathrm{GC}, \mathrm{n}=2)$, que continuou recebendo a dieta a que estava adaptado; grupo insulina (GI, $n=2)$, que além da dieta normal recebeu a administração subcutânea de insulina NPH (Humulin ${ }^{\circledR}$ N), na dose de $0,25 \mathrm{UI} / \mathrm{kg}$ de peso vivo a cada 12 horas durante os 3 dias de experimento; grupo jejum (GJ, $n=2)$, que foi submetido a jejum também por 3 dias, recebendo somente água, e o grupo jejum e insulina (GJI, $n=2)$, que foi submetido ao jejum e administração de insulina, conforme grupos anteriores.

Foram realizadas coletas de sangue durante os 3 dias do experimento, no período médio entre as duas refeições, para análise dos níveis de glicose e uréia, sendo que a partir das amostras do dia 3 também foi verificado o nível de insulina, através do método imunoensaio de eletroquimioluminescência.
No final do terceiro dia do experimento, antes dos animais receberem alimentação, foi realizada outra coleta de sangue para análise dos níveis de proteína total, albumina, beta-hidroxibutirato, triacilglicerídeos, colesterol, magnésio e fósforo, através de reações colorimétricas $\left(\mathrm{LABTEST}^{\circledR}\right.$ ) quantificadas em espectrofotômetro, e fator de crescimento semelhante a insulina tipo I (IGF-I) pelo método de radioimunoensaio.

Os animais foram submetidos diariamente a um exame clínico, no qual verificou-se freqüência cardíaca, respiratória, ruminal e temperatura retal.

\section{RESULTADOS}

Os resultados das análises sangüíneas para glicose e uréia durante os 3 dias de experimento, são demonstrados na Tabela 1, enquanto que, na Tabela 2, são descritos os valores obtidos com as análises sangüíneas realizadas ao final do terceiro dia para os outros marcadores analisados.

Durante o período experimental, não foram observadas alterações dos sinais vitais, verificados pelo exame clínico, bem como não foram observados sinais característicos de cetose.

\section{DISCUSSÃO}

Como pode ser observado nas Tabelas 1 e 2, não houve variações relevantes nos marcadores analisados entre os grupos, demonstrando uma grande capacidade de adaptação dos animais às variações nutricionais de curto prazo, como a deste experimento, visto que ovinos e bovinos adultos parecem ser resistentes a restrição alimentar, provavelmente devido a reservas ruminais de nutrientes [7]. Já em experimentos em que o tempo de exposição a diferentes níveis alimentares é maior, observam-se variações significativas em diversos marcadores metabólicos [2].

Apesar do nível de insulina ter variado marcadamente entre os grupos, não foi observada alteração no nível de glicose, degradação proteíca ou lipídica, como seria esperado em situações de hipo ou hiperinsulinemia [4], sendo que as pequenas variações observadas entre os grupos podem ter sido mascaradas pelo baixo número de animais utilizados neste experimento.

Kosior-Korzecka et al. [5], quando submeteram ovelhas a um jejum de 4 dias, observaram uma redução dos níveis de IGF-I, o que não pode ser observado neste experimento quando os grupos são 
Schneider A., Schwegler E., Goulart M.A., Roos T.B., Rabassa V.R., Burklet Del Pino F.G., Pfeifer L.F.M. \& Corrêa M.N. Efeito do jejum e da administração de insulina sobre os parâmetros...

Tabela 1. Valores médios ( \pm desvio padrão) dos marcadores glicose e uréia ao longo dos 3 dias de experimento conforme o grupo experimental (GC - grupo controle; GI - grupo insulina; GJ grupo jejum e GJI - grupo jejum mais insulina).

\begin{tabular}{|c|c|c|c|c|c|c|}
\hline \multirow[t]{2}{*}{ Grupo } & \multicolumn{2}{|c|}{ Dia 1} & \multicolumn{2}{|c|}{ Dia 2} & \multicolumn{2}{|c|}{ Dia 3} \\
\hline & $\begin{array}{l}\text { Glicose } \\
\text { (mg/dL) }\end{array}$ & $\begin{array}{l}\text { Uréia } \\
(\mathrm{mg} / \mathrm{dL})\end{array}$ & $\begin{array}{l}\text { Glicose } \\
(\mathrm{mg} / \mathrm{dL})\end{array}$ & $\begin{array}{l}\text { Uréia } \\
(\mathrm{mg} / \mathrm{dL})\end{array}$ & $\begin{array}{l}\text { Glicose } \\
(\mathrm{mg} / \mathrm{dL})\end{array}$ & $\begin{array}{l}\text { Uréia } \\
\text { (mg/dL) }\end{array}$ \\
\hline $\mathrm{GC}$ & $\begin{array}{c}49,36 \pm \\
3,41\end{array}$ & $\begin{array}{c}18,94 \pm \\
3,16\end{array}$ & $\begin{array}{c}32,32 \pm \\
2,05\end{array}$ & $\begin{array}{c}24,63 \pm \\
5,04\end{array}$ & $\begin{array}{c}30,55 \pm \\
5,46\end{array}$ & $\begin{array}{c}22,87 \pm \\
0,30\end{array}$ \\
\hline GI & $\begin{array}{c}39,87 \pm \\
3,18\end{array}$ & $\begin{array}{c}19,89 \pm \\
8,88\end{array}$ & $\begin{array}{c}22,19 \pm \\
6,82\end{array}$ & $\begin{array}{c}24,95 \pm \\
1,43\end{array}$ & $\begin{array}{c}25,40 \pm \\
2,27\end{array}$ & $\begin{array}{c}25,05 \pm \\
1,73\end{array}$ \\
\hline GJ & $\begin{array}{c}50,64 \pm \\
12,51\end{array}$ & $\begin{array}{c}31,49 \pm \\
9,78\end{array}$ & $\begin{array}{c}23,47 \pm \\
1,82\end{array}$ & $\begin{array}{c}35,96 \pm \\
2,56\end{array}$ & $\begin{array}{c}25,40 \pm \\
1,36\end{array}$ & $\begin{array}{c}29,36 \pm \\
4,06\end{array}$ \\
\hline GJI & $\begin{array}{c}50,16 \pm \\
4,55\end{array}$ & $\begin{array}{c}23,94 \pm \\
1,50\end{array}$ & $\begin{array}{c}18,81 \pm \\
1,59\end{array}$ & $\begin{array}{c}25,53 \pm \\
0,90\end{array}$ & $\begin{array}{c}28,30 \pm \\
20,01\end{array}$ & $\begin{array}{c}24,31 \pm \\
4,14\end{array}$ \\
\hline
\end{tabular}

Tabela 2. Valores médios ( \pm desvio padrão) dos marcadores metabólicos analisados conforme o grupo experimental (GC - grupo controle; GI - grupo insulina; GJ - grupo jejum e GJI - grupo jejum mais insulina).

\begin{tabular}{|l|c|c|c|c|}
\hline Marcadores & GC & GI & GJ & GJI \\
\hline Proteína total (g/L) & $70,10 \pm 1,40$ & $75,60 \pm 12,70$ & $29,50 \pm 25,00$ & $68,80 \pm 3,80$ \\
\hline Albumina (g/L) & $32,90 \pm 0,10$ & $30,00 \pm 0,60$ & $36,40 \pm 2,40$ & $31,60 \pm 2,10$ \\
\hline Beta-hidroxibutirato (mg/dL) & $9,70 \pm 0,04$ & $12,28 \pm 3,89$ & $8,14 \pm 2,09$ & $9,03 \pm 1,20$ \\
\hline Triacilglicerídeos (mg/dL) & $26,07 \pm 2,58$ & $15,76 \pm 0,01$ & $24,25 \pm 12,00$ & $11,52 \pm 9,43$ \\
\hline Colesterol (mg/dL) & $60,64 \pm 5,77$ & $76,38 \pm 5,77$ & $62,97 \pm 15,67$ & $75,22 \pm 15,67$ \\
\hline Magnésio (mg/dL) & $2,63 \pm 0,13$ & $2,75 \pm 0,77$ & $2,64 \pm 0,26$ & $1,91 \pm 0,01$ \\
\hline Fósforo (mg/dL) & $9,93 \pm 3,44$ & $8,27 \pm 0,69$ & $10,98 \pm 4,52$ & $6,32 \pm 0,32$ \\
\hline IGF-I (ng/mL) & $\begin{array}{r}167,00 \pm \\
24,04\end{array}$ & $\begin{array}{r}126,00 \pm \\
26,87\end{array}$ & $\begin{array}{r}113,50 \pm \\
3,54\end{array}$ & $\begin{array}{r}116,50 \pm \\
14,84\end{array}$ \\
\hline Insulina ( $\mu \mathrm{UI} / \mathrm{mL})$ & $3,75 \pm 0,49$ & $11,20 \pm 4,38$ & $\begin{array}{c}1,40 \pm 0 \\
0,40 \pm 0\end{array}$ \\
\hline
\end{tabular}


comparados separadamente. Porém, quando consideramos apenas 2 grupos, os submetidos e os não submetidos ao jejum, observamos uma maior variação do nível de IGF-I nos grupos submetidos ao jejum $(115,00 \pm 8,98 \mathrm{ng} / \mathrm{mL})$ em relação aos não submetidos $(151,50 \pm 27,45 \mathrm{ng} / \mathrm{mL})$.

A ausência de sinais clínicos, característicos de cetose, concorda com o observado em um estudo realizado com indução de síntese de corpos cetônicos [3], no qual os animais permaneciam em jejum por um período semelhante, e não demonstraram sinais de cetose.

\section{CONCLUSÕES}

Ovelhas confinadas, adaptadas a um padrão alimentar, são capazes de resistir a variações de curto prazo no padrão alimentar, não apresentando mudanças consideráveis nos níveis dos marcadores metabólicos ou sinais clínicos.

\section{REFERÊNCIAS}

1 Atti N.C., Kayouli M., Mahouachi A., Guesmi \& M. Doreau. 2002. Effect of a drastic and extended underfeeding on digestion in Barbary ewe. Animal Feed Science and Technology. 100: 1-14.

2 Caldeira R.M., Belo A.T., Santos C.C., Vazques M.I. \& Portugal A.V. 2007. The effect of long-term feed restriction and over-nutrition on body condition score, blood metabolites and hormonal profiles in ewes. Small Ruminant Research. 68: 242-255.

3 Emmanuel B. \& Kennelly J.J. 1984. Effect of propionic acid on ketogenesis in lactating sheep fed restricted rations or deprived of food. Journal of Dairy Science. 67: 344-350.

4 González F.H.D. \& Silva S.C. 2006. Bioquímica clínica de glicídeos. In: Introdução à bioquímica clínica veterinária. 2 ed. Porto Alegre: Editora da UFRGS, pp.153-210.

5 Kosior-Korzecka U., Bobowiec R. \& Lipecka C.Z. 2006. Fasting-induced changes in ovulation rate, plasma leptin, gonadotropins, GH, IGF-I and insulin concentrations during oestrus in ewes. Journal of Veterinary Medicine. 53: 511.

6 Ramin A.G., Asri S. \& Majdani R. 2005. Correlations among serum glucose, beta-hydroxybutyrate and urea concentrations in non-pregnant ewes. Small Ruminant Research. 57: 265-269.

7 Tatman W.R., Judkins M.B., Krysl L.J. \& Moss G.E. 1991. Gastrointestinal digesta passage and fermentation patterns associated with restricted intake of a low quality forage in ewes. Small Ruminant Research. 4: 393-399. 